\title{
MODELING OF OPERATING PROCEDURES IN A FOREST COMPARTMENT UNDER FUZZY DYNAMIC NATURE WORKING CONDITIONS
}

\author{
Konstantin Rukomojnikov ${ }^{1^{*}}$ \\ ${ }^{1}$ Volga State University of Technology , Russia
}

In this paper the principles and calculations of stretched in time graphic-analytical models of the operating network are proposed. They allow you to determine the sequence of logging opera-tions at logging sites within the forest compartment, to reduce nonmechanical delay and increase technological efficiency of harvesting operations with fuzzy dynamic natural-production conditions. Search algorithm minimal cost flow developed to solve the proposed graphic-analytical model. It allows to justify the amount of expenses and calculate a maximum bandwidth of arcs between the fictitious source and drain of graphical model. The model differs complex use of two main charac-teristics of the process of logging operations: productivity and labor costs are presented as individu-al capacities of graph arcs.

In order to solve the suggested graphic model, we developed the search algorithm of mini-mum cost flux, providing grounds for the typical expenditures and calculating the carrying capacity between the false source and runoff within the structure of suggested graphic models, different in two core characteristics of the logging process: productivity and labour inputs, represented as the carrying capacity of the graph arcs. The suggested graphical-analytic approach represents a certain task type of graph theory, according to which the transportation of flux along the dynamic opera-tional network arcs reduces their carrying capacity. Every arc denotes analogous technological op-erations within the analysed time period. This is the distinctive feature of the suggested approach.

The suggested design and calculation principles for time expanded graphical-analytical models of the operational network enable to provide rationale for the logging consequence and technological operations in fuzzy dynamic nature environment of a forest compartment, reduce operational downtime and enhance the efficiency of forest harvesting operations.

Key words: Forest compartment, Logging, Graph theory, Flow of minimum cost, Fuzzy dynamic natural production conditions

\section{INTRODUCTION}

It is well-known that selecting a set of machines when forming a park of timber harvesting equipment at a forest engineering enterprise should be based on the approach when the effective average of all the machines included into a system equals or divisible by the productivity of the leading machine. However, the nature working conditions in every harvesting area are significantly different from the analogous mean values within the enterprise. Any deviation of the nature working conditions in every harvesting area from the main values, characterizing the total felling-area resources of the enterprise result in inconsistent operation of the whole harvesting system. This problem may be partially mitigated, when a few areas are harvested in one forest compartment $[05,06,07,08,09,10]$ based on sequencing feasibility and harvesting technique applied to each of them.

It should be taken into consideration, that in real working environment of harvesting areas such parameters as production of harvesting machines and prime costs of transportation and timber processing operations in ever-changing conditions of the environment can't be certain in advance.

Presentation of the technological processes sequence in the forest compartment in the form of a graphic- analytical model brings a researcher to a time independent and dynamic flow problem in fuzzy dynamic nature working conditions of the environment, which doesn't presume instant pass of the whole flow on the arcs of a graph.

The research aims to provide rationale to the operating procedures carried out in a forest compartment with integrated account taken of special factors relating to technological characteristics of the all the harvesting areas within the compartment.

\section{LITERATURE REVIEW}

Flow optimization in transportation is revealed in [01, 02, 03]. In [04] the author suggests a solution to the problem of the maximum flow, in which the transportation parameters are regarded as fuzzy triangular numbers, the addition, subtraction and comparison tasks for fuzzy triangular numbers are solved.

The research described above gives an idea on the possibilities of making calculations in fuzzy conditions of a transportation network. However, this research cannot be applied to managing the operating procedures of wood cutting operations without due consideration of the sector-specific issues. 


\section{MATERIALS AND METHODS}

In order to achieve the set goal, we developed the following graph options used to carry out all kinds of technological processes of harvesting operations. For example, in Fig. 1 there is a time expanded dynamic graph, characterizing a technological process of forest compartment felling including all the processing operations of a technological process on a forest swath. The graph is drawn by the formation of a separate copy of every vertex $\mathrm{X}_{\mathrm{i}} \in \mathrm{X}$ in every period under review $\theta \in \mathrm{T}$. A vertex set $X_{p}$ of a graph $G_{p}$ is set as $X_{p}=\left\{\left(x_{i}, \theta\right):\left(X_{i}, \theta\right) \in X \times T\right\}$. The arcs set $\widetilde{A}_{\mathrm{p}}$ is represented by arcs, formed by every couple "vertex-time" $\left(x_{i}, \theta\right) \in X_{p}$ into every couple "vertex-time" $\left(x_{j}, \theta\right)$ and $\left(x_{j}, \theta+\tau_{i j}(\theta)\right)$. Thus, $x \in \Gamma\left(x_{i}\right)$, and $\theta+\tau_{i j}(\theta) \leq p$.

The transportation capacity of $\widetilde{v}\left(x_{i}, x_{j}, \theta, \theta+\tau_{i j}(\theta)\right)$, connecting the couple "vertex-time" $\left(x_{i}, \theta\right)$ and $\left(x_{j}, \theta+\tau_{i j}(\theta)\right)$ equal $\infty$, while the transportation capacity of $\widetilde{v}\left(x_{i}, x_{j}, \theta, \theta\right)$, connecting the couples "vertex-time" $\left(x_{i}, \theta\right)$ and $\left(x_{i j}, \theta\right)$ equal $\widetilde{V}_{i j}(\theta)$ and can be calculated by means of the analysis of man-hour valued defined in the graph $\tilde{f}_{i j}(\theta)$ used to carry out certain operations.

Vertex $S$ represents a false source, while vertex $T$ represents a false runoff. Every process procedure is represented in the graph as intermediate vertex $x_{N_{h} \ell \theta}$ , located between a source and a runoff. Where $N$ - is the name of the process operation, $h$ - is the sequence number of an operation in the overall sequence of the process flow in a forest compartment; $I$ - is a number of the analyzed harvesting area, $\theta-$ is the number of the analyzed time period. Thus, for example, vertices $x_{B_{1} 11}, x_{B_{1} 21}, \ldots, x_{B_{1} k 1}, \ldots, x_{B_{1} g 1}$ refer to the processing operations (felling, limbing, bucking), carried out on the forest swaths of every of $g$ analysed harvesting areas for the first time period, vertices $x_{T_{2} 12}, x_{T_{2} 22}, \ldots, x_{T_{2} k 2}, \ldots, x_{T_{2} g 2}$ refer to a skid logging transportation to the loading point carried out within a second time period, vertices $x_{B_{3}^{\prime} 11}$ refer to an operation of timber hauling from the loading point in the first time period, etc.

The transportation capacity of every false arch originating from a source is limited by the deposit of harvested timber on every of $\mathrm{g}$ wood-cutting area located within a forest compartment. This indicator on a graph may be represented taking into consideration fuzzy transportation capacity of arcs, and be denoted by $\widetilde{V}_{1}, \widetilde{V}_{2}, \ldots, \widetilde{V}_{k} \ldots, \widetilde{V}_{g}$ Since this source is a false vertex, then the cost parameters of timber transportation along the arc going out equal 0.

The arcs of this graph drawn between the vertices, indicating the operations of one time period, characterize the movement of raw timber on the stages of technological process. The flow of the process along these arcs is characterized by fuzzy flow capacity of arcs, denoting the productivity of harvesting machines and equipment, which are in direct relations to labour inputs $(\tilde{f})$ necessary to complete every operation, as well as fuzzy variable costs $(\tilde{C})$. The latter include fuel cost, piecework wages, etc. Fuzzy variable costs are fixed when flows move between the operations of a technological process. The movement cost of $\tilde{\mathrm{C}}\left(x_{i}, x_{j}, \theta, \theta\right)$ flow unit along the graph arcs connecting the time-vertex couple $\left(\mathbf{x}_{\mathrm{i}}, \boldsymbol{\theta}\right)$ and $(x, \theta)$ equal $\widetilde{\mathrm{C}}_{i j}(\theta)$.

Thus, it is possible to take into consideration both fixed and variable costs necessary to carry out operations analyzed in a graph. The arcs drawn between the vertices referring to the same operation but carried out in different time periods, refer to the raw timber somehow excluded from the subsequent state of the technological process in the time periods considered earlier. Thus, for example, fixed costs $(Z)$, which are fixed when the flow moves from one time period into the next, include depreciation costs, planned maintenance and machine repair costs, wages costs provided that employees are paid based on hourly basis, etc. The movement cost of $Z\left(x_{i}, x_{j}, \theta, \theta+\tau_{i j}(\theta)\right)$ flow unit along the graph arcs connecting the time-vertex couple $\left(x_{i}, \theta\right)$ and $\left(x_{j}, \theta+\tau_{i j}(\theta)\right)$ equal $Z_{i j}(\theta)$.

The problem of a maximum flow at a minimum cost in the suggested fuzzy dynamic network of process operations of timber harvesting in a forest compartment may be formulated using the following mathematical relations:

1. It is necessary to define the minimum route of timber movement along the arcs of a dynamic operational network in the suggested number of periods.

$$
\sum_{\theta=1}^{p} \sum_{\left(x_{i}, x_{j}\right) \in \tilde{A}}\left(\tilde{C}_{i j} \cdot \tilde{\xi}_{i j}(\theta)+Z_{i j} \cdot \min \left\{1 ; \tilde{\xi}_{i j}(\theta)\right\}\right) \rightarrow \min
$$

2. The maximum volume of flow $\tilde{v}$ taken for the $p$ periods of time equals to the flow originating from the source in $p$ periods of time.

$$
\sum_{\theta=1}^{p} \sum_{x_{j} \in X}\left[\tilde{\xi}_{s j}(\theta)-\tilde{\xi}_{j s}\left(\theta-\tau_{j s}(\theta)\right)\right]-\tilde{v}(p)=\tilde{0}
$$

3. The value of flow $\tilde{\xi}_{i i}$, included into the vertex $x_{i}$ in the time period $\left(\theta-\tau_{j i}\right)$ equals the number of flow units $\tilde{\xi}_{i j}$, going out of a vertex $x_{i}$ in time period $\theta$;

$$
\sum_{x_{j} \in X}\left[\tilde{\xi}_{i j}(\theta)-\tilde{\xi}_{j i}\left(\theta-\tau_{j i}(\theta)\right)\right]=\tilde{0}
$$$$
x_{i} \neq s, t ; \theta \in T \text {. }
$$

This conditions should be met for every vertex $x_{i}$ with no regard to false vertices of a source and run off;

4. The maximum value of the flow $\tilde{v}$, passing through the graph arcs in $p$ time periods, equals the flow, entering the runoff in the same time period.

$\sum_{\theta=1}^{p} \sum_{x_{j} \in X}\left[\tilde{\xi}_{t j}(\theta)-\tilde{\xi}_{j t}\left(\theta-\tau_{j t}(\theta)\right)\right]-\tilde{v}(p)=\tilde{0}$ 
5. In order to provide grounds for the flow value, it is necessary to take into consideration the fact that the total work efforts used to carry out the same technological operations of every $(\theta)$ time period should not exceed the maximum working time $(m)$ of this period. Thus, the value of flow $\left(\xi_{(i=b) j}^{N}(\theta)\right)$, going along the arc $\left(x_{i=b}, x_{j}\right)$ in the analyzed time period $(\theta)$, should correspond to the following equation:

6.

$$
0 \leq \tilde{\xi}_{(i=b) j}^{N}(\theta) \leq \frac{m(\theta)-\sum_{i \in[1 ; b) \cup(b ; g]} \sum_{x_{j} \in X} \tilde{f}_{i j}^{N}(\theta) \cdot \tilde{\xi}_{i j}^{N}(\theta)}{\tilde{f}_{(i=b) j}^{N}(\theta)}
$$

This equation should be true for all the time periods and all graph vertices, besides the source and the arcs, connecting different time periods.

$$
\begin{aligned}
& \forall\left(x_{i}, x_{j}\right) \in \tilde{A}(\theta) ; \theta \in T ; \\
& x_{i} \neq s ;\left(x_{i}, x_{j}\right) \neq\left(x_{N_{h} \ell \theta} ; x_{N_{h} \ell(\theta+1)}\right)
\end{aligned}
$$

where $b$ - is the ordinal number of the analysed arc $(1 \leq b \leq g) ; m$ - is the maximum working time of the analysed period measured in man-hours; $\tilde{f}_{i j}^{N}(\theta)$ - is labour inputs used to process or transport a timber unit when carrying out $N$-operation of the technological process in $\theta$-time period, $\mathrm{h} / \mathrm{m}^{3} ; \tilde{\xi}_{i j}^{N}(\theta)$ - is the flow value, transported from vertex $i$ into $\theta-$ is the time period for carrying out $N$-operations of the technological process, $\mathrm{m}^{3}$.

One of the parameters, restricting the capacity of arcs is the productivity $\Pi(\theta)$. In our case by productivity we mean the scope of work, which can be completed before the end of the analyzed time period. In the course of calculation, as a result of cargo traffic movement along the graph arcs, there is a reduction of time $m^{*}(\theta)$ left before the completion of the analysed stage, which equals:

$$
m^{*}(\theta)=m(\theta)-\sum_{i=1}^{g} \sum_{x_{i} \in X} \tilde{f}_{i j}^{N}(\theta) \cdot \tilde{\xi}_{i j}^{N}(\theta)
$$

Thus, the labour inputs used to carry out this or that operation of a technological process and machine and mechanisms performance are interconnected:

$$
\Pi_{i j}^{N}(\theta)=\frac{m^{*}(\theta)}{f_{i j}^{N}(\theta)} ; f_{i j}^{N}(\theta)=\frac{m^{*}(\theta)}{\Pi_{i j}^{N}(\theta)}
$$

Under fuzzy natural environment the productivity and labour inputs may be represented as fuzzy triangular numbers:

$$
\begin{aligned}
\widetilde{\Pi}_{i j}(\theta) & =\left(\Pi_{i j}(\theta) ; \Pi_{i j}{ }^{L}(\theta) ; \Pi_{i j}{ }^{R}(\theta)\right) \\
\tilde{f}_{i j}(\theta) & =\left(f_{i j}(\theta) ; f_{i j}{ }^{L}(\theta) ; f_{i j}{ }^{R}(\theta)\right)
\end{aligned}
$$

The initial values of the left and right borders of fuzzy triangular numbers are set by the experts. These indicators are interrelated and can be calculated:

$$
\begin{aligned}
& f_{i j}^{N^{L}}(\theta)=f_{i j}^{N}(\theta)-\frac{m^{*}(\theta)}{\Pi_{i j}^{N}(\theta)+\Pi_{i j}^{N^{R}}(\theta)} \\
& f_{i j}^{N^{R}}(\theta)=\frac{m^{*}(\theta)}{\Pi_{i j}^{N}(\theta)-\Pi_{i j}^{N^{L}}(\theta)}-f_{i j}^{N}(\theta)
\end{aligned}
$$

The formulas presented make is possible to get the inverse relation:

$$
\begin{aligned}
\Pi_{i j}^{N}(\theta) & =\frac{m^{*}(\theta)}{f_{i j}^{N}(\theta)-f_{i j}^{N^{L}}(\theta)}-\Pi_{i j}^{N}(\theta) \\
\Pi_{i j}^{N}{ }^{L}(\theta) & =\Pi_{i j}^{N}(\theta)-\frac{m^{*}(\theta)}{f_{i j}^{N}(\theta)+f_{i j}^{N^{R}}(\theta)}
\end{aligned}
$$

The labour inputs, characterising the inverse arcs equal as follows:

$$
f_{j i}^{N}(\theta)=\frac{m_{j i}^{* \text { обр }}(\theta)}{\prod_{j i}^{N}(\theta)}
$$

Where $m_{j i}^{* \text { oбp }}$ is the time of technological operation, which forms the inverse arc on the graph.

The limiting values of the triangular numbers, denoting labour inputs may be calculated based on the relations.

$$
\begin{aligned}
& f_{j i}^{N^{L}}(\theta)=f_{j i}^{N}(\theta)-\frac{m_{j i}^{* \text { обр }}(\theta)}{\Pi_{j i}^{N}(\theta)+\Pi_{j i}^{N^{R}}(\theta)} \\
& f_{j i}^{N^{R}}(\theta)=\frac{m_{j i}^{* 0 \sigma \mathrm{p}}(\theta)}{\Pi_{j i}^{N}(\theta)-\Pi_{j i}^{N^{L}}(\theta)}-f_{j i}^{N}(\theta)
\end{aligned}
$$

A detailed numerical example of solving the problem is considered in [10].

\section{RESULTS AND DISCUSSIONS}

In order to solve the suggested graphic model we developed the search algorithm of minimum cost flux, providing grounds for the typical expenditures and calculating the carrying capacity between the false source and runoff within the structure of suggested graphic models, different in two core characteristics of the logging process: productivity and labour inputs, represented as the carrying capacity of the graph arcs. The suggested graphical-analytic approach represents a certain task type of graph theory, according to which the transportation of flux along the dynamic operational network arcs reduces their carrying capacity. Every arc denotes analogous technological operations within the analysed time period. 
This is the distinctive feature of the suggested approach. The suggested design and calculation principles for time expanded graphical-analytical models of the operational network enable to provide rationale for the logging consequence and technological operations in fuzzy dynamic nature environment of a forest compartment, reduce operational downtime and enhance the efficiency of forest harvesting operations.

\section{REFERENCES}

1. Bershtein L.S., Belyakov S.L., Bozhenyuk A.V. and Rozenberg I.N. 2014. Routing in the conditions of uncertainty with the use of fuzzy temporal graphs. Life Science Journal. T. 11. № 7s.: 182-186.

2. Bozhenyuk A., Gerasimenko E. (2013). Methods for maximum and minimum cost flow determining in fuzzy conditions. World Applied Sciences Journal. T. 22. № SPL.ISSUE 2: 76-81.

3. Bozhenyuk A., Gerasimenko E. (2014). Flows finding in networks in fuzzy conditions. Studies in Fuzziness and Soft Computing. T. 313.: 269-291.

4. Bozhenyuk A., Gerasimenko, E. and Rozenberg, I. (2012). The task of minimum cost flow finding in transportation networks in fuzzy conditions. Proceedings of the 10th International FLINS Conference Concept on Uncertainty Modeling in Knowledge Engineering and Decision Making Word Scientific, Istanbul, Turkey: 354-359;

5. Rukomojnikov K.P., (2007). Substantiation of a technique of account the basic technological parameters within the planning quarter borders. Moscow state forest university bulletin — Lesnoy vestnik. №4(53): 96-102.
6. Rukomojnikov K.P., (2013) Technical and technological aspects of progressive cutting forest compartment with combined reforestation. World Applied Sciences Journal. Volume 24, Issue 11: 1429-1434.

7. Rukomojnikov K.P., (2014). Graphic-analytical approach to rationale of placing secondary road on territory of forest compartment. Modern problems of science and education. №6: http://www.science-education.ru/120-16418

8. Rukomojnikov K.P., (2014). Justification sequences to perform basic transport operations of logging operations during harvesting within the forest compartment. Modern problems of science and education. №6: http://www.science-education.ru/120-16401

9. Rukomojnikov K.P., (2015). Structuring of loading points and main skid road in the forest compartment. Modern problems of science and education. №1: http://www.science-education. ru/121-19239

10. Rukomojnikov K.P., (2015). Structuring of loading points and main skid road in conditions of existing road network in forest compartment. Journal of Applied Engineering Science. №13, 3, 326: 167 - 174

11. Rukomojnikov K.P., (2016). The choice of rational technology and justification of parameters of the quarterly development of forest areas, Yoshkar-Ola: Volga StateUniversity of Technology. 